\title{
Acute Cytokine Response During Breast Cancer Surgery: Potential Role of Dexamethasone and Lidocaine and Relationship with Postoperative Pain and Complications - Analysis of Three Pooled Pilot Randomized Controlled Trials
}

This article was published in the following Dove Press journal: Journal of Pain Research

\author{
Sandra AS van den Heuvel, (D) ' \\ Selina El van der Wal, (D) \\ Ewald M Bronkhorst, (iD ${ }^{2}$ \\ Michiel C Warlé, (iD ${ }^{3}$ \\ May Ronday, (D) ${ }^{4}$ Judith Plat, (D) ${ }^{4}$ \\ Nens van Alfen, (iD) \\ Leo $A B$ Joosten, (iD ${ }^{6}$ \\ Jos GC Lerou, (iD) \\ Kris CP Vissers, (iD) \\ Monique AH Steegers ${ }^{7}{ }^{7}$ \\ 'Department of Anesthesiology, Pain and \\ Palliative Medicine, Radboud University Medical \\ Centre, Nijmegen, the Netherlands; \\ ${ }^{2}$ Department of Health Evidence, Radboud \\ University Medical Centre, Nijmegen, the \\ Netherlands; ${ }^{3}$ Division of Vascular and Transplant \\ Surgery, Department of Surgery, Radboud \\ University Medical Centre, Nijmegen, the \\ Netherlands; ${ }^{4}$ Department of Anesthesiology, \\ Alexander Monro Breast Cancer Hospital, \\ Bilthoven, the Netherlands; ${ }^{5}$ Donders Institute \\ for Brain Cognition and Behavior, Department of \\ Neurology, Radboud University Medical Centre, \\ Nijmegen, the Netherlands; ${ }^{6}$ Department of \\ Internal Medicine and Radboud Centre for \\ Infectious Diseases, Radboud University Medical \\ Centre, Nijmegen, the Netherlands; \\ ${ }^{7}$ Department of Anesthesiology, Pain and \\ Palliative Medicine, Amsterdam University \\ Medical Centre, Amsterdam, the Netherlands
}

Correspondence: Sandra AS van den Heuvel

Department of Anesthesiology, Pain and Palliative Medicine, Radboud University Nijmegen Medical Center, Internal mail 630, P.O. Box 9101, Nijmegen 6525 GA, the Netherlands

Tel +3I 2436I5244

Email Sandra.vandenHeuvel@radboudumc.nl
Purpose: An imbalance in perioperative cytokine response may cause acute pain and postoperative complications. Anesthetic drugs modulate this cytokine response, but their role in non-major breast cancer surgery is unclear. In an exploratory study, we investigated whether intravenous lidocaine and dexamethasone could modulate the cytokine response into an anti-inflammatory direction. We also evaluated interrelationships between cytokine levels, pain scores and postoperative complications. Our goal is to develop multimodal analgesia regimens optimizing outcome after breast cancer surgery.

Patients and Methods: Forty-eight patients undergoing a lumpectomy were randomly assigned to placebo or lidocaine $\left(1.5 \mathrm{mg} \cdot \mathrm{kg}^{-1}\right.$ followed by $\left.2 \mathrm{mg} \cdot \mathrm{kg}^{-1} \cdot \mathrm{hour}^{-1}\right)$ supplemented by dexamethasone zero, 4 or $8 \mathrm{mg}$, yielding six groups of eight patients. Interleukin (IL)-1 $\beta$, IL-1Ra, IL-6, IL-10 levels and pain scores were measured at baseline and four hours postoperatively. We assessed postoperative complications occurring within 30 days. We noted persistent pain and infections as potential immune-related complications (PIRC). We used multiple regression to disentangle the effects of the individual study drugs (given by their partial regression coefficients (b)). Odds ratios (OR) estimated the link between pain scores and complications.

Results: Dexamethasone $8 \mathrm{mg}$ increased IL-10 ( $\mathrm{b}=12.70$ (95\% $\mathrm{CI}=8.06-17.34), P<0.001)$. Dexamethasone $4 \mathrm{mg}$ and $8 \mathrm{mg}$ decreased the ratio $\mathrm{IL}-6 / \mathrm{IL}-10(\mathrm{~b}=-2.60$ ( -3.93 to -1.26$)$, $P<0.001$ and $\mathrm{b}=-3.59$ ( -5.04 to -2.13 ), $P<0.001$, respectively). We could not show modulatory effects of lidocaine on cytokines. High pain scores were linked to the occurrence of PIRC's $(\mathrm{OR}=2.028$ (1.134-3.628), $P=0.017$ ). Cytokine levels were not related either to acute pain or PIRC. Conclusion: Dexamethasone modulated the perioperative cytokine response into an antiinflammatory direction. An overall lidocaine effect was not found. Patients with higher pain scores suffered from more 30-day PIRCs. Cytokine levels were not associated with pain or more postoperative complications, even not with PIRC. Larger studies in breast cancer surgery are needed to confirm these explorative results.

Keywords: acute pain, anesthetic agents, immune response, lumpectomy, perioperative outcomes

\section{Plain Language Summary}

Cytokines are small proteins that are crucial in controlling the activity of the body's immune system. The body's response to an injury or other trigger like surgery is called "inflammation". 
Surgery is a cornerstone of breast cancer treatment, but induces the release of pro-inflammatory and anti-inflammatory cytokines. An imbalance in the pro-inflammatory direction results in more pain and more morbidity after an operation. This may contribute to the potential negative impact of breast cancer surgery on the quality of life in up to half of the patients. Anesthetic drugs used during surgery, such as lidocaine and dexamethasone, may however counteract the cytokine imbalance beneficially.

\section{What Is Unknown?}

- The impact of dexamethasone and lidocaine on cytokine responses is unclear for patients undergoing conservative breast cancer surgery, like lumpectomy without axillary lymph node dissection.

- The interrelationships between cytokine levels, pain scores and complications after lumpectomy are unknown.

We investigated 48 patients undergoing breast cancer surgery to answer two questions. Do lidocaine and/or dexamethasone steer the immune response into a beneficial anti-inflammatory direction? Does an interrelationship exist between cytokine levels, pain scores and postoperative complications?

What This Article Adds

- The immune response of patients receiving dexamethasone $4 \mathrm{mg}$ or $8 \mathrm{mg}$ goes into an anti-inflammatory direction.

- Patients experiencing less pain suffer less from postoperative complications.

Results for lidocaine were inconclusive and we could not demonstrate a link between patient's cytokine levels and complications. Larger studies are thus needed to confirm our results and to help optimizing perioperative outcomes.

\section{Introduction}

Surgery is a cornerstone of breast cancer treatment, but induces a systemic inflammatory response characterized by the release of cytokines. Proinflammatory cytokines like interleukin (IL)-1 $\beta$, IL-6 and tumor necrosis factor alpha are released after tissue injury. Anti-inflammatory cytokines, like IL-10 and IL-1Ra, are also released to restore homeostasis. ${ }^{1}$ An imbalance between pro- and anti-inflammatory cytokines activates an inflammatory cascade resulting in increased postsurgical morbidity. Increased levels of IL-6 and ratio of IL-6/IL-10 are proven predictors for developing infectious complications after surgery. ${ }^{2-4}$

Postoperative pain, chronic pain and postoperative infectious complications contribute to the overall negative impact of breast cancer surgery on the quality of life in up to half of the patients. ${ }^{5,6}$ Acute postoperative pain, which is a predictive factor for the development of chronic pain, ${ }^{7,8}$ may be related to the inflammatory responses after surgery. ${ }^{9}$ This may help to explain why higher pain scores after surgery contribute to postoperative complications, especially health care-associated infections. ${ }^{10}$

Various pharmacological regimens proved to counteract the surgery-induced proinflammatory response beneficially, thus reducing morbidity after operation. ${ }^{11}$ Dexamethasone, given in a high dose of up to $100 \mathrm{mg}$ before cardiac surgery with cardiopulmonary bypass, shifted the cytokine profile into the anti-inflammatory direction. ${ }^{12}$ Intravenous (IV) lidocaine attenuates the hyperinflammatory response after tissue injury, thus reducing pain and improving outcomes. ${ }^{13,14}$

For patients undergoing breast cancer surgery, and specifically in conservative surgery like lumpectomy without axillary lymph node dissection, the impact of dexamethasone and IV lidocaine on cytokine responses is unclear. The question thereby arises if a single low dose of dexamethasone could be effective enough. Four mg is commonly administered for its anti-emetic effect and $8 \mathrm{mg}$ for its additional analgesic effect. ${ }^{15}$ Furthermore, the interrelationships between cytokine levels, pain scores and postoperative complications after lumpectomy have not yet been investigated. Understanding immune-mediated mechanisms underlying acute pain and complications could help develop multimodal analgesia regimens and optimize outcome after breast cancer surgery.

Therefore, we performed an explorative study including patients undergoing a lumpectomy for breast cancer. First, we aimed to evaluate whether a low dose of dexamethasone or IV lidocaine modulates the early cytokine response into an anti-inflammatory direction. Second, we aimed to confirm the link between higher pain scores and 30-day surgical complications. Third, we aimed to investigate whether a proinflammatory-oriented cytokine balance is associated with higher pain scores and immune-mediated surgical complications.

\section{Patients and Methods}

We consecutively performed three double blinded randomized controlled trials at the Alexander Monro Hospital, a non-teaching hospital (10 hospital beds) specialized in breast cancer care (Bilthoven, The Netherlands). The Institutional Review Board approved all study protocols and documents. The study was registered prior to patient enrollment at the European Clinical Trial Register (EudraCT 2012-00222270; Principal Investigator: Prof. K.C.P. Vissers; Date of registration: November 15, 2013). The protocol can be accessed after a reasonable request at protocols.io 
(https://www.protocols.io/edit/targeting-the-inflammatoryresponse-after-breast-c-bc56iy9e). Procedures followed were in accordance with the Declaration of Helsinki and adhere to the applicable Consolidated Standards of Reporting Trials (CONSORT) guidelines. Participating patients gave written informed consent.

\section{Patients}

We assessed potential participants for eligibility during the preoperative anesthesia consultation. Female patients aged older than 18 years undergoing primary breast cancer surgery without axillary lymph node dissection were eligible for inclusion. Exclusion criteria were: allergy to amide type local anesthetics, myocardial ischemia within the previous six months, hypokalemia $\left(<3.5 \mathrm{mmol} \cdot \mathrm{L}^{-1}\right)$, renal impairment (creatinine clearance $<60 \mathrm{~mL} \cdot \mathrm{min}^{-1} \cdot 1.73 \mathrm{~m}^{-2}$ ) or liver function impairment (international normalized ratio $>1.8$ ), pregnancy or breastfeeding, use of anti-arrhythmic drugs, chronic opioid use, a history of chronic pain, corticosteroid use, and the inability to provide written informed consent.

\section{Patient Groups}

In the three consecutive studies, patients received no dexamethasone or dexamethasone $4 \mathrm{mg}$ or dexamethasone $8 \mathrm{mg}$ before induction of general anesthesia. In each of the studies, patients were randomly assigned in a 1:1 ratio to one of two groups (IV lidocaine or IV placebo) using a sealed envelope with blocks of eight.

An external pharmacy delivered the blinded study medication to the anesthetic department in identically appearing $50 \mathrm{~mL}$ syringes. Lidocaine $1.5 \mathrm{mg} \cdot \mathrm{kg}^{-1}$ or placebo (saline $0.9 \%$ in the same volume) was administered as a bolus in ten minutes before induction of anesthesia, followed by a continuous infusion of IV lidocaine at $2 \mathrm{mg} \cdot \mathrm{kg}^{-1} \cdot$ hour $^{-1}$ or placebo until one hour after end of surgery. The lidocaine dosing regimen is consistent with most references, but the optimal dosing is unknown. ${ }^{13}$ Potential adverse events caused by IV lidocaine were noted. They were reported by patients or observed by site staff monitoring patient's vital signs.

\section{Measurements}

There were four measuring points: at baseline, when patients arrived on the operation theater complex before surgery ( $\mathrm{t} 0)$, admission on post-anesthesia care unit (PACU) (t1), 4 hours after end of surgery (t2), and 30 days after surgery.
Pain scores were assessed using the Numeric Rating Scale $\left(\right.$ NRS $0=$ no pain, NRS $10=$ worst pain imaginable) ${ }^{16}$ NRS was recorded at $\mathrm{t} 0, \mathrm{t} 1$, and $\mathrm{t} 2$.

Cytokine levels of IL-6, IL-10, IL-1 $\beta$ and IL-1Ra were measured at $\mathrm{t} 0$ and $\mathrm{t} 2$. Ethylenediaminetetraacetic anticoagulated blood was centrifugated immediately after withdrawal at $2000 \times \mathrm{g}$ for ten minutes at $4^{\circ} \mathrm{C}$, after which plasma was stored at $-80^{\circ} \mathrm{C}$. Cytokines were analyzed batch-wise by Simplex Assays ${ }^{\mathrm{TM}}$ according to the manufacturer's instructions as previously described. ${ }^{17}$ Baseline levels of low abundance cytokines, including IL-6, IL-10 and IL-1 $\beta$, proved to be below the lower limit of quantification by standard enzyme-linked immunosorbent assays. The Ella microfluidic analyzer (Protein Simple, San Jose, CA, USA) was therefore used to assess cytokine concentrations in the $\mathrm{fg} \cdot \mathrm{mL}^{-1}$ to low $\mathrm{pg} \cdot \mathrm{mL}^{-1}$ range. ${ }^{18}$

We classified any medical adverse outcome occurring between admission and 30 days after surgery according to the Clavien Dindo Classification (CDC) of surgical complications. The CDC systematically scores postoperative complications in five grades and is a preferred method for outcome assessment after surgery. ${ }^{19}$ A complication with a potential immune-mediated pathway, ie an infection or persistent postsurgical pain, was noted as a potential immune-related complication (PIRC). ${ }^{3,9,20}$ The study physician searched retrospectively for complications and then classified them according to CDC. A medical researcher, who was not involved in the study or patient's care, checked the records to ensure completeness of data.

\section{Perioperative Management}

Acetaminophen 1 gr PO was given one hour before operation. We induced anesthesia with IV propofol and sufentanil and maintained it with continuous infusion of IV propofol supplemented with sufentanil according to clinical needs. The total dose of sufentanil at end of surgery was recorded. Intraoperative monitoring included pulse oximetry, capnography, electrocardiography and non-invasive blood pressure measurement. IV diclofenac $75 \mathrm{mg}$ was administered at the end of surgery unless there was a contraindication for non-steroidal anti-inflammatory drugs.

Patients remained at the PACU for at least one hour until discontinuation of the study medication. Nurses assessed postoperative nausea and vomiting and the Aldrete score per standard protocols. Patients with NRS $\geq 4$ at admission on the PACU received IV piritramide 2.5 $\mathrm{mg}$ at ten minutes intervals until $\mathrm{NRS} \leq 3$. Clonidine $75 \mu \mathrm{g}$ 
was given at ten minutes intervals when, despite administration of piritramide, the NRS remained $\geq 4$ on condition that the heart rate was $\geq 80 \mathrm{bpm}$ and systolic blood pressure was $\geq 120 \mathrm{mmHg}$. Postoperative pain management on the ward consisted of acetaminophen 1 gram four times daily, diclofenac $50 \mathrm{mg}$ three times daily and piritramide $0.1 \mathrm{mg} \cdot \mathrm{kg}^{-1}$ six times daily as needed.

After discharge patients received standard care. One week post-surgery a follow-up visit was scheduled with a nurse and the surgeon. Thereafter, patients received regular clinical care by their radiotherapist, surgeon or nurse, depending on their oncologic plan. Postoperative complications were documented in patients' records.

\section{Statistical Analysis}

Our study is based upon the analysis of pooled data obtained in three consecutive randomized controlled trials. As this was an explorative study, no formal sample size calculation was made. Our results should, therefore, be regarded as explorative and hypothesis-generating.

Results for all variables are given as mean (SD) [minimum-maximum], unless stated otherwise. The differences between plasma levels of cytokines at $\mathrm{t} 2$ and those at $\mathrm{t} 0$ are given as $\Delta \mathrm{IL}-6, \Delta \mathrm{IL}-10, \Delta \mathrm{IL}-1 \beta$ and $\Delta \mathrm{IL}-1 \mathrm{Ra}$. The balance between pro- and anti-inflammatory cytokines is described by the ratio of IL-6/IL-10 and the ratio of IL-1 $\beta$ / IL-1Ra. The differences between these ratios at $\mathrm{t} 2$ and those at $\mathrm{t} 0$ are given as $\Delta \mathrm{IL}-6 / \mathrm{IL}-10$ and $\Delta \mathrm{IL}-1 \beta / \mathrm{IL}-1 \mathrm{Ra}$.

Multiple linear regression analysis was used to evaluate the null hypothesis that lidocaine or dexamethasone had no effect on $\Delta \mathrm{IL}-6, \Delta \mathrm{IL}-10, \Delta \mathrm{IL}-1 \beta, \Delta \mathrm{IL}-1 \mathrm{Ra}, \Delta \mathrm{IL}-6 /$ IL-10 and $\Delta \mathrm{IL}-1 \beta / \mathrm{IL}-1 \mathrm{Ra}$. Descriptive analysis preceded formal statistical analysis. Based on a striking pattern in the dataset, we introduced "post hoc" the duration of surgery as an independent variable.

Univariate binary logistic regression models using pooled data were estimated to evaluate the potential association between NRS at $\mathrm{t} 2$ and the occurrence of any complication according to CDC or a PIRC. The association between the cytokine levels and their ratios at t0 and $\mathrm{t} 2$ on one hand, and the occurrence of a PIRC on the other hand, was evaluated similarly.

A Spearman's Rho correlation was used to determine if NRS at $\mathrm{t} 2$ was related to each of the cytokine levels at $\mathrm{t} 0$ and $\mathrm{t} 2$.

Statistical analysis was performed using IBM SPSS Statistics V25.0 and GraphPad Prism V8.0 (GraphPad Software). $P<0.05$ was considered statistically significant.

\section{Results}

We analyzed data for 48 patients with eight patients in each of six groups (Figure 1).

The six groups were similar in respect of physical and clinical characteristics, except surgery duration (Table 1). All patients underwent breast-conserving surgery with a sentinel node biopsy. The intraoperative and direct postoperative course was uneventful. All patients could be discharged from the PACU one hour after admission (Aldrete score $\geq 13$ ).

We supply patients' characteristics of pooled lidocaine groups and pooled dexamethasone groups in the Supplementary Table S1 and all raw cytokine levels in the Supplementary Table S2. IL-1 $\beta$ levels at baseline were lower in the study with dexamethasone $8 \mathrm{mg}$ than those in the other two studies.

\section{Pain and Its Treatment}

Pain scores and pain treatment are summarized in Table 1. At t $1,94 \%$ of the patients had NRS $\leq 4$ and $96 \%$ at $\mathrm{t} 2$. Opioid and diclofenac use was similar among patient groups during and after operation. Rescue drug clonidine was not administered in the third trial where dexamethasone $8 \mathrm{mg}$ was given alone or combined with IV lidocaine.

\section{Postoperative Complications}

A total of 23 postoperative complications occurred in 22 (45.8\%) patients (Table 1). Sixteen patients $(33 \%$ of the total) had a CDC grade I complication. Four patients (8\%) had a CDC grade II, and two patients had a CDC grade III complication. The groups were similar in respect of complications.

Eight patients suffered from a PIRC: persistent pain $(n=3)$, mucositis (1), surgical site infection treated with antibiotics (2), and abscess treated with antibiotics and surgery (2). Fourteen patients suffered from the following complications: hematoma (6), seroma (3), nausea (3), constipation (1), light headedness (1), and a transient ischemic attack (1). One of these fourteen patients suffered from two CDC grade I complications.

\section{Effects of Dexamethasone and Lidocaine on Postoperative Cytokine Levels}

Figures 2 and 3 reveal patterns in the individual observations. Observations above the zero line in Figures 2 and 3 are increases in cytokine levels or their ratios between t0 and $\mathrm{t} 2$. 


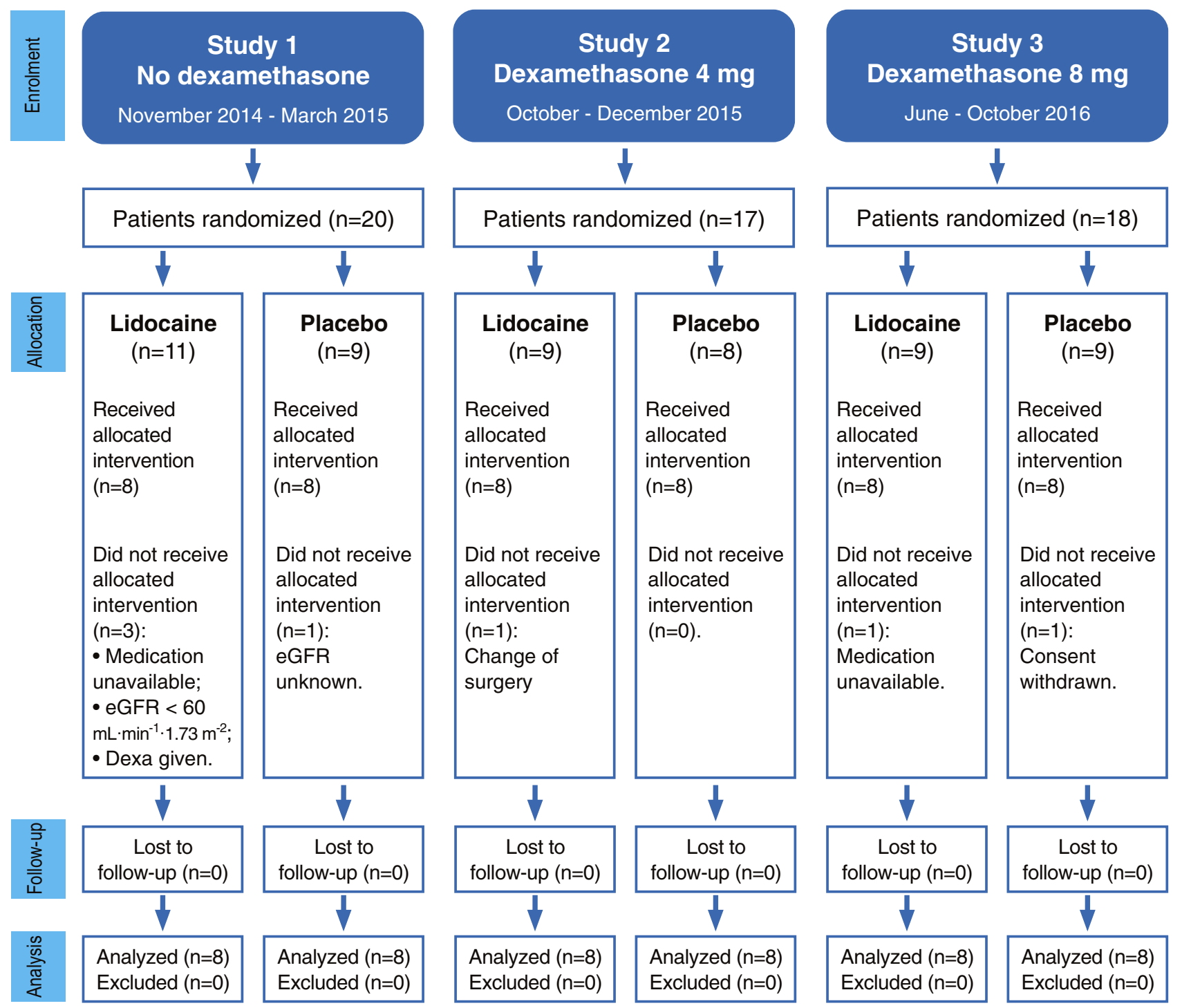

Figure I Consolidated Standards of Reporting Trials (CONSORT) flow chart of the three consecutive randomized controlled trials.

In most patient groups the levels of IL-6 and IL-10 (Figure $2 \mathrm{~A}$ and $\mathrm{B}$ ) and IL-1Ra (Figure 3B) seemed to increase after surgery. In contrast, IL- $1 \beta$ levels (Figure $3 \mathrm{~A}$ ) appeared not to change. Lidocaine had little or no effect on the cytokine levels and their ratios in comparison with placebo (Figures $2 \mathrm{~A}$ and $\mathrm{C}, 3 \mathrm{~A}-\mathrm{C}$ ), except that it seemed to increase IL-10 levels when combined with dexamethasone 8 $\mathrm{mg}$ (Figure 2B). Dexamethasone 4 and $8 \mathrm{mg}$ appeared to reverse $\Delta \mathrm{IL}-6 / \mathrm{IL}-10$ (Figure $2 \mathrm{C}$ ) but seemed to have no effect on IL-6 (Figure 2A), IL-1 $\beta$ (Figure 3A), and IL-1Ra (Figure 3B) levels. Dexamethasone $8 \mathrm{mg}$ appeared to increase the IL-10 level (Figure 2B). Figures $2 \mathrm{C}$ and $3 \mathrm{C}$ suggest that a longer duration of surgery increased $\triangle \mathrm{IL}-6 /$ IL-10 but decreased $\Delta$ IL-1 $\beta /$ IL-1Ra.
Multiple regression confirms our descriptive analysis and untangles the separate effects of lidocaine, dexamethasone $4 \mathrm{mg}$, dexamethasone $8 \mathrm{mg}$ and duration of surgery (Table 2). An overall effect of lidocaine on measured cytokines was not found. There is only suggestive evidence that dexamethasone $4 \mathrm{mg}$ increased IL-10 level ( $P=0.045$; positive "b" in Table 2 means increase). Dexamethasone $4 \mathrm{mg}$ decreased IL-6/IL-10 $(P<0.001$; negative "b" means decrease). Dexamethasone $8 \mathrm{mg}$ increased the IL-10 level $(P<0.001)$ and decreased IL-6/IL-10 $(P<0.001)$. As a "post hoc" independent variable the duration of surgery increased postoperative IL-6 levels $(\mathrm{P}=0.026)$ and IL-6/IL-10 $(\mathrm{P}=0.04)$, but decreased IL-1 $\beta / I L-1$ Ra $(\mathrm{P}=0.016)$.). Eg the result for surgery time in Table 2 means that the average 
Table I Patients' Perioperative Characteristics in the Three Consecutive Randomized Controlled Trials

\begin{tabular}{|c|c|c|c|c|c|c|}
\hline & \multicolumn{2}{|c|}{ RCT I: No Dexamethasone } & \multicolumn{2}{|c|}{ RCT 2: Dexamethasone $4 \mathrm{mg}$} & \multicolumn{2}{|c|}{ RCT 3: Dexamethasone 8 mg } \\
\hline & Placebo, $n=8$ & Lidocaine, $n=8$ & Placebo, $n=8$ & Lidocaine, $n=8$ & Placebo, $n=8$ & Lidocaine, $n=8$ \\
\hline Age (yr) & 55 (13) [34-73] & 59 (II) [42-75] & $61(12)[43-75]$ & $55(8)$ [43-68] & 55 (8) [47-67] & $61(11)[47-80]$ \\
\hline BMI $\left(\mathrm{kg} \mathrm{m}^{-2}\right)$ & $26(5)[20-34]$ & $28(6)[20-36]$ & $25(5)[20-33]$ & $23(4)[|9-3|]$ & $25(4)[2 I-35]$ & $24(4)[20-32]$ \\
\hline \multicolumn{7}{|l|}{ ASA physical status (n) } \\
\hline 1 & 2 & 4 & 3 & 6 & 5 & 5 \\
\hline 2 & 6 & 4 & 5 & 2 & 3 & 3 \\
\hline \multicolumn{7}{|l|}{ Tumour classification (n) } \\
\hline Carcinoma in situ & 2 & 2 & 2 & 1 & 1 & 1 \\
\hline TI & 4 & 5 & 5 & 4 & 6 & 5 \\
\hline $\mathrm{T} 2$ & 2 & 1 & 1 & 3 & 1 & 2 \\
\hline \multicolumn{7}{|l|}{ Node classification (n) } \\
\hline No & 7 & 8 & 7 & 6 & 5 & 7 \\
\hline $\mathrm{NI}$ & 1 & 0 & 1 & 2 & 3 & 1 \\
\hline Surgery duration (min) & $109(42)[39-152]$ & $87(35)[35-156]$ & $76(18)[58-110]$ & $92(36)$ [24-133] & 65 (19) [39-92] & $68(24)[36-109]$ \\
\hline $\begin{array}{l}\text { Sufentanil }\left(\mu \mathrm{kg}^{-1}\right. \\
\left.\mathrm{hr}^{-1}\right)\end{array}$ & $\begin{array}{l}0.32(0.09)[0.20- \\
0.45]\end{array}$ & $\begin{array}{l}0.25(0.13)[0.13- \\
0.54]\end{array}$ & $\begin{array}{l}0.29(0.09)[0.17- \\
0.43]\end{array}$ & $\begin{array}{l}0.26(0.15)[0.12- \\
0.60]\end{array}$ & $\begin{array}{l}0.31(0.09)[0.17- \\
0.44]\end{array}$ & $\begin{array}{l}0.29(0.16)[0.11- \\
0.52]\end{array}$ \\
\hline \multicolumn{7}{|l|}{ NRS } \\
\hline At $t 0$ & $0.9(1.1)[0-3]$ & $0.1(0.4)[0-1]$ & $0.3(0.5)[0-1]$ & $0.8(1.4)[0-4]$ & $0(0)[0-0]$ & $0.4(1.1)[0-3]$ \\
\hline At $\mathrm{tl}$ & I.6 (2.1) $[0-5]$ & $1.5(1.5)[0-4]$ & $1.3(1.8)[0-5]$ & $1.4(1.5)[0-4]$ & $1.5(2.1)[0-6]$ & $0.3(0.7)[0-2]$ \\
\hline At $\mathrm{t} 2$ & $2.4(1.2)[0-7]$ & $1.8(1.5)[0-4]$ & $1.9(1.1)[0-4]$ & $2.1(1.7)[0-4]$ & $2.6(0.9)[2-4]$ & $2.4(1.2)[0-4]$ \\
\hline \multicolumn{7}{|l|}{ Diclofenac (n) } \\
\hline $0 \mathrm{mg}$ & 1 & 0 & 1 & 0 & 0 & 1 \\
\hline $75 \mathrm{mg}$ & 6 & 6 & 7 & 7 & 8 & 7 \\
\hline $125 \mathrm{mg}$ & 1 & 2 & 0 & I & 0 & 0 \\
\hline Piritramide (mg) & $9.7(6.0)[0-20]$ & $8.1(5.8)[0-20]$ & $8.8(4.8)[5-20]$ & $8.4(6.3)[0-20]$ & $9.4(3.7)[5-17.5]$ & $10.0(4.4)[5-20]$ \\
\hline \multicolumn{7}{|l|}{ Clonidine (n) } \\
\hline $0 \mu \mathrm{g}$ & 6 & 5 & 7 & 7 & 8 & 8 \\
\hline $75 \mu \mathrm{g}$ & 1 & 2 & 1 & 1 & 0 & 0 \\
\hline $150 \mu g$ & 1 & 1 & 0 & 0 & 0 & 0 \\
\hline \multirow{2}{*}{\multicolumn{7}{|c|}{$\begin{array}{l}\text { 30-Day complications } \\
\text { (N) }\end{array}$}} \\
\hline & & & & & & \\
\hline CDC I & 2 & 2 & 5 & 4 & 3 & 0 \\
\hline CDC II & 0 & 1 & 0 & 1 & 0 & 2 \\
\hline CDC IIIb & 1 & 0 & 0 & 0 & 0 & 1 \\
\hline
\end{tabular}

Notes: Data are mean (SD) [minimum-maximum], number of patients $(n)$ or number of complications $(N)$. At to $=$ baseline, $t \mathrm{l}=$ direct postoperative, and $t 2=4$ hours after surgery.

Abbreviations: RCT, randomized controlled trial; BMI, body mass index; ASA, American Society of Anesthesiologists; T, tumor staging; NRS, Numeric Rating Scale; CDC, Clavien Dindo Classification.

increase in IL-6 level was $0.162 \mathrm{pg} \cdot \mathrm{mL}^{-1}$ per minute or 9.72 $\mathrm{pg} \cdot \mathrm{mL}^{-1}$ per hour of surgery time, independently from the other independent variables.

IL-1Ra increased from $268 \mathrm{pg} \cdot \mathrm{mL}^{-1}$ to $8075 \mathrm{pg} \cdot \mathrm{mL}^{-1}$ in one patient of the dexamethasone $8 \mathrm{mg}$-lidocaine group (Figure 3B). As this outlier was not an influential observation, it was not excluded from regression analysis.

\section{Relationships Between Cytokine Levels, Pain Scores and Postoperative Complications}

Patients who experienced higher pain scores at $\mathrm{t} 2$ seemed to be prone to suffer from any complication according to CDC within 30 days after surgery (OR $=1.464,95 \%$ $\mathrm{CI}=0.941-2.278 ; P=0.091)$ (Table 3 ). A statistically significant association exists between having postoperative 

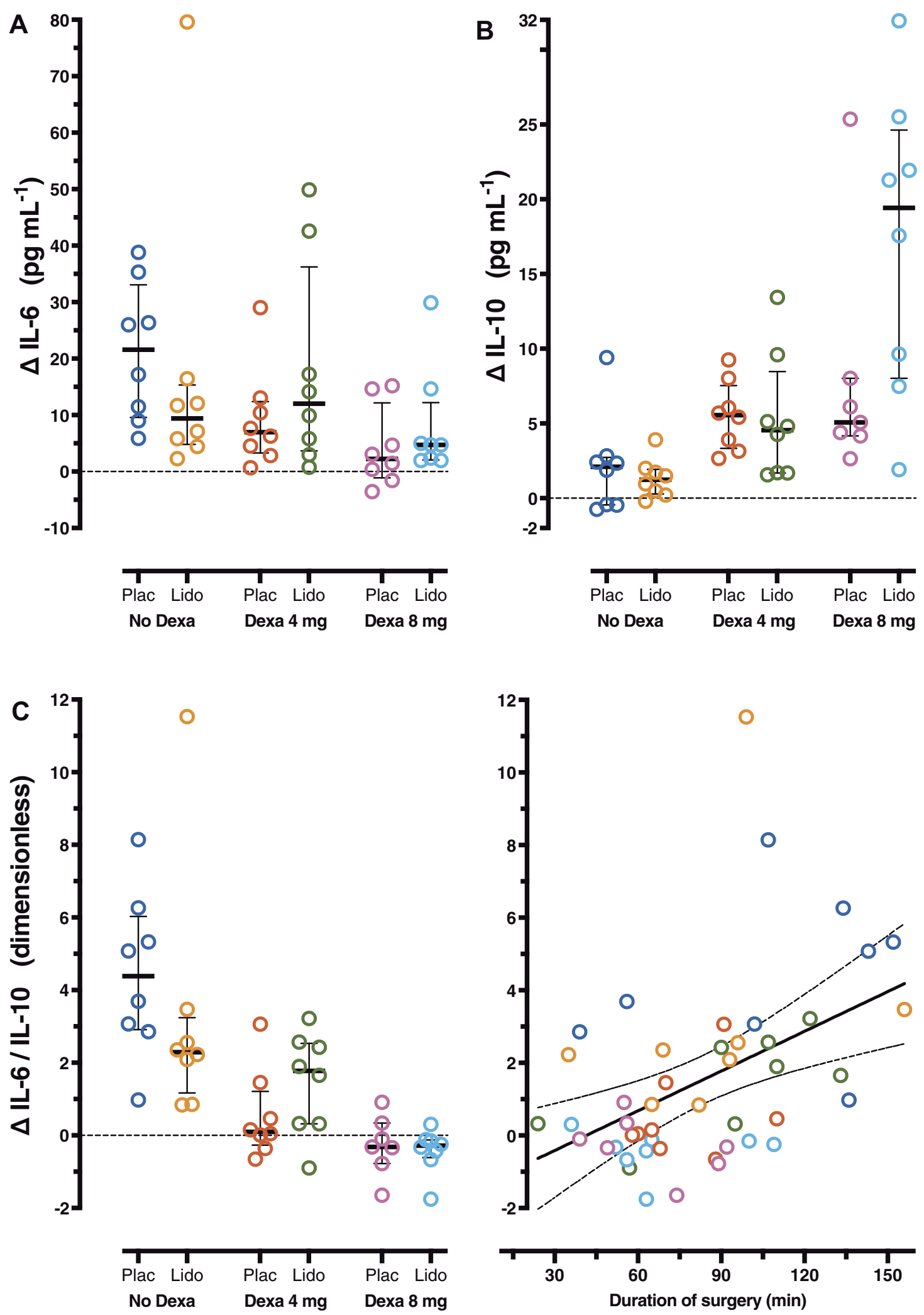

Figure 2 Difference $(\Delta)$ between interleukin (IL)-6, IL-10 plasma levels and ratio IL-6/IL-10 at baseline (t0) and those 4 hours after surgery (t2) in six groups ( $\mathrm{n}=8$ per group). Each symbol represents one patient. A symbol above or below the zero line represents an increase or decrease, respectively, from to to t2. Error bars are the 25 th75th percentiles around the medians. (A) Observations for $\Delta \mathrm{IL}-6$ and (B) for $\Delta \mathrm{IL}-10$ per group. (C) Observations for the ratio $\Delta \mathrm{IL}-6 / \mathrm{IL}-10$ per group and linear regression of the pooled observations on the duration of surgery. The full regression line is accompanied with dotted lines showing its $95 \%$ confidence limits. There is one missing value in the group dexamethasone $8 \mathrm{mg}$ with placebo for $\Delta \mathrm{IL}-10$ and $\Delta \mathrm{IL}-6 / \mathrm{LL}-10$. Plac, placebo; Dexa, dexamethasone; Lido, lidocaine. 

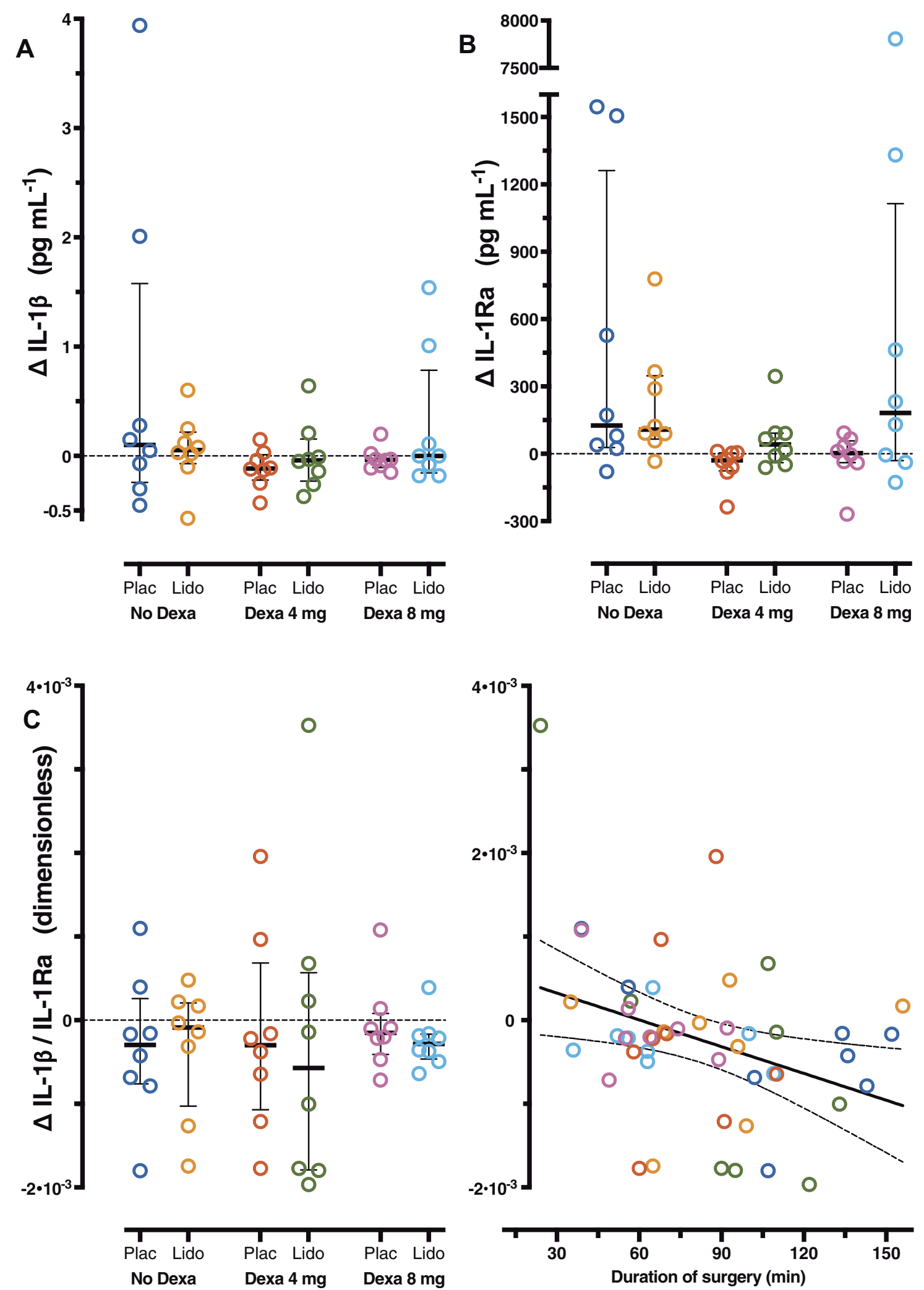

Figure 3 Difference $(\Delta)$ between interleukin (IL)- I $\beta$, IL-I Ra plasma levels and ratio IL-I $\beta / I L-I R a$ at baseline ( $t 0)$ and those 4 hours after surgery (t2) in six groups $(n=8$ per group). Each symbol represents one patient. A symbol above or below the zero line represents an increase or decrease, respectively, from t0 to t2. Error bars are the 25 th75th percentiles around the medians. (A) Observations for $\Delta \mathrm{IL}-\mathrm{I} \beta$ and (B) for $\Delta \mathrm{IL}-\mathrm{IRa}$ per group. (C) Observations for the ratio $\Delta \mathrm{IL}-\mathrm{I} / \mathrm{IL}-\mathrm{IRa}$ per group and linear regression of the pooled observations on the duration of surgery. The full regression line is accompanied with dotted lines showing its $95 \%$ confidence limits. Plac, placebo; Dexa, dexamethasone; Lido, lidocaine. 
Table 2 The Effects (Column "b") of Lidocaine, Dexamethasone 4 mg, Dexamethasone 8 mg and Surgery Duration on the Levels of Interleukins and Their Ratios After Surgery

\begin{tabular}{|c|c|c|c|c|c|c|c|c|c|}
\hline & \multicolumn{3}{|l|}{$\Delta \mathrm{IL}-6$} & \multicolumn{3}{|l|}{$\Delta \mathrm{IL}-10$} & \multicolumn{3}{|c|}{$\Delta \mathrm{IL}-6 / \mathrm{IL}-10$} \\
\hline & b & $(95 \% \mathrm{Cl})$ & $\mathbf{P}$ & b & $(95 \% \mathrm{Cl})$ & $\mathbf{P}$ & b & $(95 \% \mathrm{Cl})$ & $\mathbf{P}$ \\
\hline Intercept & 2.03 & $(-14.14-18.19)$ & 0.802 & -4.74 & $(-11.41-1.94)$ & 0.160 & 2.03 & $(-0.07-4.12)$ & 0.057 \\
\hline Lidocaine & 3.02 & $(-5.30-11.34)$ & 0.468 & 2.61 & $(-0.86-6.08)$ & 0.137 & -0.12 & $(-1.21-0.97)$ & 0.824 \\
\hline Dexamethasone $4 \mathrm{mg}$ & -3.54 & $(-13.91-6.82)$ & 0.494 & 4.37 & $(0.10-8.65)$ & 0.045 & -2.60 & $(-3.93--1.26)$ & $<0.001$ \\
\hline Dexamethasone $8 \mathrm{mg}$ & -8.04 & $(-19.15-3.07)$ & 0.152 & 12.70 & $(8.06-17.34)$ & $<0.001$ & -3.59 & $(-5.04--2.13)$ & $<0.001$ \\
\hline \multirow[t]{4}{*}{ Surgery time (min) } & 0.162 & $(0.020-0.303)$ & 0.026 & 0.053 & $(-0.005-0.111)$ & 0.074 & 0.019 & $(0.001-0.037)$ & 0.040 \\
\hline & & $\mathrm{R}^{2}=0.227$ & & & $R^{2}=0.449$ & & & $R^{2}=0.526$ & \\
\hline & \multicolumn{3}{|l|}{$\Delta \mathrm{IL}-\mathrm{I} \beta$} & \multicolumn{3}{|c|}{$\Delta I L-I R a$} & \multicolumn{3}{|c|}{$\Delta \mathrm{IL}-\mathrm{I} \beta / \mathrm{IL}-\mathrm{IRa}$} \\
\hline & b & $(95 \% \mathrm{Cl})$ & $P$ & b & $(95 \% \mathrm{Cl})$ & $P$ & b & $(95 \% \mathrm{Cl})^{*}$ & $P$ \\
\hline Intercept & 0.83 & $(0.03-1.64)$ & 0.043 & 124 & $(-1210-1458)$ & 0.852 & 0.88 & $(-0.20-1.97)$ & 0.108 \\
\hline Lidocaine & -0.08 & $(-0.50-0.34)$ & 0.699 & 367 & $(-319-1053)$ & 0.287 & -0.11 & $(-0.67-0.45)$ & 0.701 \\
\hline Dexamethasone $4 \mathrm{mg}$ & -0.49 & $(-1.01-0.03)$ & 0.062 & -338 & $(-1193-517)$ & 0.430 & -0.07 & $(-0.76-0.63)$ & 0.843 \\
\hline Dexamethasone $8 \mathrm{mg}$ & -0.39 & $(-0.94-0.17)$ & 0.167 & 268 & $(-649-1184)$ & 0.559 & -0.21 & $(-0.96-0.53)$ & 0.571 \\
\hline \multirow[t]{2}{*}{ Surgery time (min) } & -0.004 & $(-0.011-0.003)$ & 0.229 & 0.420 & $(-11.23-12.07)$ & 0.942 & -0.012 & $(-0.021--0.002)$ & 0.016 \\
\hline & & $R^{2}=0.097$ & & & $R^{2}=0.070$ & & & $R^{2}=0.134$ & \\
\hline
\end{tabular}

Notes: Results are intercept and partial regression coefficients (b) with their $95 \% \mathrm{Cl}$ obtained from multiple regression analysis. $*$ Results for IL-I $\beta / \mathrm{IL}-\mathrm{IRa}$ are to be multiplied by $10^{-3}$. $\Delta$, difference between the levels of $\mathrm{IL}$ and their ratios at baseline and those at 4 hours after surgery. $\mathrm{R}^{2}$, coefficient of determination.

Abbreviations: IL, interleukin; $\mathrm{Cl}$, confidence interval.

pain and the occurrence of a PIRC: the odds ratio associated with a one-point increase in NRS is $2.028(95 \%$ $\mathrm{CI}=1.134-3.628 ; P=0.017)$. This implies that patients who score an NRS of 5 have eight times the odds of having immune-related complications as those with an NRS of $2\left(2.028^{3}=8.341\right.$, where the exponent 3 is the difference between 5 and 2).

Cytokine levels showed no relation with the occurrence of a PIRC (Table 3), although there is some suggestive evidence that the preoperative ratio of IL-6/IL-10 could relate to the occurrence of a PIRC $(\mathrm{OR}=2.918,95 \%$ $\mathrm{CI}=0.848-10.047 ; P=0.089$ ).

No correlation was found between NRS at $\mathrm{t} 2$ and cytokine levels at t0 or t 2 . The low $P$-value $(P=0.078)$, for the correlation between postoperative IL-10 levels and pain scores at t2 might be regarded as "indicative". However, no correction for the number of tests was done (Supplementary Table S3).

\section{Discussion}

This prospective explorative study in 48 patients undergoing a lumpectomy for breast cancer has several findings, but the small sample size in each group precludes drawing firm conclusions. Dexamethasone $4 \mathrm{mg}$ or $8 \mathrm{mg}$, but not IV lidocaine, modulated the early cytokine response into an anti-inflammatory direction. Raw cytokines levels were not clearly related to postoperative pain scores or the occurrence of a PIRC. However, there was suggestive evidence that a more pro-inflammatory cytokine balance at baseline could result in more PIRC's.

Despite the small number of patients, we may be more confident in the evidence supporting a link between higher pain scores noted 4 hours postoperatively and PIRC in our homogenous group of patients as our finding accords with previous research. ${ }^{10}$ In a cohort of 1014 patients undergoing a broad spectrum of surgeries, higher pain scores or unacceptable pain after surgery were associated with more 30-day complications, especially health care-associated infections. $^{10}$

Anesthetic agents may aid to improve postoperative outcomes $^{21,22}$ by counteracting the neuroendocrine stress response to surgery or through a direct effect on immune cells. The choice of anesthetic agents can even be crucially important for patients with pre-existing immune dysfunction or tumor burden. ${ }^{21,22}$ However, the impact of lidocaine or dexamethasone, which have different immune-modulating effects on cytokine responses, has not been investigated after lumpectomy.

IV lidocaine is known to reduce a hyperinflammatory response, ${ }^{13}$ and to decrease proinflammatory cytokines in abdominal surgery. ${ }^{23,24}$ Under the circumstances of our study no overall effect of lidocaine on postoperative 
Table 3 Relationships Between Cytokine Levels, Pain Scores and Postoperative Complications

\begin{tabular}{|c|c|c|c|}
\hline & & Odds Ratio (95\% Cl) & $\mathbf{P}$ \\
\hline \multirow[t]{2}{*}{ NRS } & $\mathrm{t} 2$ & $\mathrm{I} .464(0.94 \mathrm{I}-2.278) \dagger$ & 0.091 \\
\hline & t2 & $2.028(1.134-3.628) \ddagger$ & 0.017 \\
\hline \multirow[t]{2}{*}{ IL-6 } & to & $1.359(0.918-2.010)$ & 0.125 \\
\hline & t2 & $1.036(0.992-1.082)$ & 0.113 \\
\hline \multirow[t]{2}{*}{ IL-I0 } & to & $0.690(0.207-2.301)$ & 0.546 \\
\hline & $\mathrm{t} 2$ & $1.053(0.963-\mid .151)$ & 0.255 \\
\hline \multirow[t]{2}{*}{ IL-6/IL-I0 } & to & $2.918(0.848-10.047)$ & 0.089 \\
\hline & t2 & I. 196 (0.917-I.559) & 0.186 \\
\hline \multirow[t]{2}{*}{ IL-I $\beta$} & to & $0.32 \mid(0.007-\mid 4.874)$ & 0.561 \\
\hline & $\mathrm{t} 2$ & $0.802(0.228-2.822)$ & 0.731 \\
\hline \multirow[t]{2}{*}{ IL-IRa } & to & $1.003(0.999-1.007)$ & 0.136 \\
\hline & $\mathrm{t} 2$ & $1.000(0.999-1.001)$ & 0.940 \\
\hline \multirow[t]{2}{*}{ IL-I $\beta / I L-I R a *$} & to & $0.521(0.167-1.623)$ & 0.261 \\
\hline & t2 & I.25I (0.60I-2.605) & 0.549 \\
\hline
\end{tabular}

Notes: Odds ratios with $95 \%$ confidence intervals $(\mathrm{Cl})$ obtained from univariate binary logistic regressions estimating the association between pain scores or cytokine levels on one hand and the occurrence of a 30-day complication on the other. For pain scores, which were obtained with Numeric Rating Scale (NRS) at 4 hours after operation ( $t 2)$ the dependent variable was the occurrence of any complication according to the $\mathrm{CDC}^{\dagger}$ or only a potentially immune-related complication ${ }^{\ddagger}$ (PIRC). For cytokine levels and two of their ratios at baseline $(\mathrm{t} 0)$ and $\mathrm{t} 2$, the dependent variable was the occurrence of a PIRC. NRS was associated with PIRC in these univariate analyses. *Results for IL-I $\beta / I L-I R a$ are to be multiplied by $10^{-3}$.

cytokine levels was found. Most likely, lumpectomy is a surgical procedure with less inflammatory stimuli, ${ }^{23}$ where lidocaine may have little effect.

Dexamethasone's anti-inflammatory effects depend on its dosage in relation to the extent of tissue trauma. After high dose corticosteroids for major abdominal or thoracic surgery, earlier reports demonstrated a shift towards an anti-inflammatory balance characterized by suppressed IL-6 and increased IL-10 levels, while effects on IL-1 and IL-1Ra levels were absent or showed a decrease. ${ }^{12,25,26}$ A lower dose, like dexamethasone $8 \mathrm{mg}$, during abdominal surgery had no effect on IL-10 levels, and reduced ${ }^{27}$ or showed no effect on IL-6 levels. ${ }^{28}$ As lumpectomy can be regarded as minimally invasive surgery, when compared with abdominal or cardiac surgery, a less pronounced increase of pro-inflammatory cytokines could be expected. Accordingly, we found a decrease in IL-10 levels and in the ratio IL-6/IL-10, but no significant effect on the levels of IL-6 and IL-1 $\beta$. Surprisingly, patients in the third pilot study had pre-existent lower levels of IL-1 $\beta$, which could have masked a potential effect of dexamethasone $8 \mathrm{mg}$ on the postoperative IL-1 $\beta$ levels in this group.
Increased IL-6 levels proved to be related to infectious complications $^{3,4}$ and higher pain levels. ${ }^{29,30}$ Also in pathological pain models, elevated expressions of IL- 6 and IL-1 $\beta$ in the spinal cord and dorsal root ganglia have been found.31,32 In our study we found a link between higher pain scores and more PIRC's, suggesting a similar underlying immune-mediated mechanism. However, we failed to find a statistically significant relation between cytokine levels and pain scores or PIRC's. Besides a small sample size, potential explanations are: the postoperative cytokine response was influenced by dexamethasone, the postoperative inflammatory response was minimal, the time of measurement was not well chosen, or the range of pain scores was too small. Interestingly, patients with a pre-existent higher IL-6 to IL-10 ratio tended to develop more postoperative complications, which can indicate a vulnerability in a subset of patients.

Our study was not powered to find a statistically significant difference in postoperative pain scores between patient groups, but a clear pattern was present. All patients, who received dexamethasone $8 \mathrm{mg}$, did not need extra clonidine as rescue analgesic. Those patients who received also lidocaine, apart from the dexamethasone $8 \mathrm{mg}$, had no pain immediately after surgery. These findings accord with those of others. ${ }^{33-36}$

Our study has important limitations that are to be avoided in future research. We performed the three studies in a consecutive order so that dexamethasone was not blindly administered (Figure 1). This may have influenced expectations and subsequently postoperative pain scores. A further limitation was the small number of patients in each group. Thus, negative results are to be interpreted with caution. Furthermore, we only measured cytokine levels measured at a single time point postoperatively. Hence, we cannot draw any firm conclusions whether the early postoperative immune imbalance could explain the acute pain and complications. Lastly, we used current literature $^{3,9,20}$ to designate not only infections but also pain as complications with a potential immune-mediated mechanism. Notwithstanding, we conceivably missed other specific immune-mediated complications which could have influenced our results.

A strength is that we performed a single-center study with a small team and a strictly protocolized environment. Consequently, inclusion rate was rather low. An extra delaying factor was that we had to switch from a standard to a more sensitive measuring method to assess very low cytokine concentrations. ${ }^{18}$ Thus, time bias cannot be ruled out. 


\section{Conclusions}

Our study suggests research subjects for future larger scale studies in patients undergoing a lumpectomy for breast cancer: (1) Confirming that dexamethasone $4 \mathrm{mg}$ or 8 $\mathrm{mg}$, but not IV lidocaine, modulates the cytokine response into an anti-inflammatory direction. These effects should be evaluated by measuring cytokine levels on several time points postoperatively and surgery duration should be included as a variable. (2) Investigating the relation between acute pain and postoperative complications, especially PIRC's. (3) Investigating the relation between 30day complications and the preoperative ratio IL-6/IL-10 or other immune-mediated mechanisms.

Perioperatively a dynamic balance exists between proand anti-inflammatory cytokines. It is still an ongoing debate about the kind of immune balance we should aim at. $^{22}$ Although larger studies are needed, we provided new pieces of information that may help developing a multimodal approach to the prevention of complications after breast cancer surgery.

\section{Abbreviations}

CDC, Clavien Dindo Classification; CI, confidence interval; IL, interleukin; IV, intravenous; NRS, Numeric Rating Scale; OR, odds ratio; PACU, post anesthesia care unit; PIRC, potential immune-related complications; SD, standard deviation.

\section{Data Sharing Statement}

The datasets generated during the current study are available from the corresponding author on reasonable request.

\section{Disclosure}

The authors report no conflicts of interest in this work.

\section{References}

1. Desborough JP. The stress response to trauma and surgery. $\mathrm{Br} J$ Anaesth. 2000;85(1):109-117. doi:10.1093/bja/85.1.109

2. Miyaoka K, Iwase M, Suzuki R, et al. Clinical evaluation of circulating interleukin-6 and interleukin-10 levels after surgery-induced inflammation. $J$ Surg Res. 2005;125(2):144-150. doi:10.1016/j. jss.2004.12.001

3. Mokart D, Merlin M, Sannini A, et al. Procalcitonin, interleukin 6 and systemic inflammatory response syndrome (SIRS): early markers of postoperative sepsis after major surgery. Br J Anaesth. 2005;94 (6):767-773. doi:10.1093/bja/aei143

4. Oka Y, Murata A, Nishijima J, et al. Circulating interleukin 6 as a useful marker for predicting postoperative complications. Cytokine. 1992;4(4):298-304. doi:10.1016/1043-4666(92)90070-8
5. Tian Y, Schofield PE, Gough K, Mann GB. Profile and predictors of long-term morbidity in breast cancer survivors. Ann Surg Oncol. 2013;20(11):3453-3460. doi:10.1245/s10434-013-3004-8

6. Verbelen H, Tjalma W, Meirte J, Gebruers N. Long-term morbidity after a negative sentinel node in breast cancer patients. Eur J Cancer Care. 2019;28:e13077.

7. Schug SA, Bruce J. Risk stratification for the development of chronic postsurgical pain. Pain Rep. 2017;2(6):e627. doi:10.1097/ PR9.0000000000000627

8. Lavand'homme P. Transition from acute to chronic pain after surgery. Pain. 2017;158(Suppl 1):S50-S54. doi:10.1097/j.pain.0000000000 000809

9. Austin PJ, Moalem-Taylor G. The neuro-immune balance in neuropathic pain: involvement of inflammatory immune cells, immune-like glial cells and cytokines. J Neuroimmunol. 2010;229(1-2):26-50. doi:10.1016/j.jneuroim.2010.08.013

10. van Boekel RLM, Warle MC, Nielen RGC, et al. Relationship between postoperative pain and overall 30-day complications in a broad surgical population: an observational study. Ann Surg. 2019;269(5):856-865.

11. Amaya F, Hosokawa T, Okamoto A, et al. Can acute pain treatment reduce postsurgical comorbidity after breast cancer surgery? A literature review. Biomed Res Int. 2015;2015:641508. doi:10.1155/2015/641508

12. El Azab SR, Rosseel PM, de Lange JJ, et al. Dexamethasone decreases the pro- to anti-inflammatory cytokine ratio during cardiac surgery. Br J Anaesth. 2002;88(4):496-501. doi:10.1093/bja/88.4.496

13. van der Wal SE, van den Heuvel SA, Radema SA, et al. The in vitro mechanisms and in vivo efficacy of intravenous lidocaine on the neuroinflammatory response in acute and chronic pain. Eur J Pain. 2016;20(5):655-674. doi:10.1002/ejp.794

14. Kranke P, Jokinen J, Pace NL, et al. Continuous intravenous perioperative lidocaine infusion for postoperative pain and recovery. Cochrane Database Syst Rev. 2015;7:CD009642.

15. Waldron NH, Jones CA, Gan TJ, Allen TK, Habib AS. Impact of perioperative dexamethasone on postoperative analgesia and sideeffects: systematic review and meta-analysis. $\mathrm{Br} J$ Anaesth. 2013;110(2):191-200. doi:10.1093/bja/aes431

16. Hjermstad MJ, Fayers PM, Haugen DF, et al. Studies comparing numerical rating scales, verbal rating scales, and visual analogue scales for assessment of pain intensity in adults: a systematic literature review. J Pain Symptom Manage. 2011;41(6):1073-1093. doi:10.1016/j.jpainsymman.2010.08.016

17. Aldo P, Marusov G, Svancara D, David J, Mor G. Simple Plex ${ }^{\mathrm{TM}}$ : a novel multi-analyte, automated microfluidic immunoassay platform for the detection of human and mouse cytokines and chemokines. Am $J$ Reprod Immunol. 2016;75(6):678-693. doi:10.1111/aji.12512

18. Ter Horst R, Jaeger M, Smeekens SP, et al. Host and environmental factors influencing individual human cytokine responses. Cell. 2016;167(4):1111-1124 e1113. doi:10.1016/j.cell.2016.10.018

19. Clavien PA, Barkun J, de Oliveira ML, et al. The Clavien-Dindo classification of surgical complications: five-year experience. Ann Surg. 2009;250(2):187-196. doi:10.1097/SLA.0b013e3181b13ca2

20. Arnaoutoglou E, Kouvelos G, Papa N, et al. Prospective evaluation of post-implantation inflammatory response after EVAR for AAA: influence on patients' 30 day outcome. Eur J Vasc Endovasc Surg. 2015;49(2):175-183. doi:10.1016/j.ejvs.2014.12.006

21. Cruz FF, Rocco PR, Pelosi P. Anti-inflammatory properties of anesthetic agents. Crit Care. 2017;21(1):67. doi:10.1186/s13054-0171645-x

22. Hsing $\mathrm{CH}$, Wang JJ. Clinical implication of perioperative inflammatory cytokine alteration. Acta Anaesthesiol Taiwan. 2015;53(1):2328. doi:10.1016/j.aat.2015.03.002

23. Ortiz MP, Godoy MC, Schlosser RS, et al. Effect of endovenous lidocaine on analgesia and serum cytokines: double-blinded and randomized trial. J Clin Anesth. 2016;35:70-77. doi:10.1016/j. jclinane.2016.07.021 
24. Yardeni IZ, Beilin B, Mayburd E, Levinson Y, Bessler H. The effect of perioperative intravenous lidocaine on postoperative pain and immune function. Anest Analg. 2009;109(5):1464-1469. doi:10.1213/ANE. 0b013e3181bab1bd

25. Sato N, Koeda K, Ikeda K, et al. Randomized study of the benefits of preoperative corticosteroid administration on the postoperative morbidity and cytokine response in patients undergoing surgery for esophageal cancer. Ann Surg. 2002;236(2):184-190. doi:10.1097/ 00000658-200208000-00006

26. Holte K, Kehlet H. Perioperative single-dose glucocorticoid administration: pathophysiologic effects and clinical implications. J Am Coll Surg. 2002;195(5):694-712. doi:10.1016/S1072-7515(02)01491-6

27. Zargar-Shoshtari K, Sammour T, Kahokehr A, Connolly AB, Hill AG. Randomized clinical trial of the effect of glucocorticoids on peritoneal inflammation and postoperative recovery after colectomy. Br J Surg. 2009;96(11):1253-1261. doi:10.1002/bjs.6744

28. Kirdak T, Yilmazlar A, Cavun S, Ercan I, Yilmazlar T. Does single, low-dose preoperative dexamethasone improve outcomes after colorectal surgery based on an enhanced recovery protocol? Double-blind, randomized clinical trial. Am Surg. 2008;74(2):160-167.

29. Lisowska B, Maldyk P, Kontny E, Michalak C, Jung L, Cwiek R. Postoperative evaluation of plasma interleukin-6 concentration in patients after total hip arthroplasty. Ortop Traumatol Rehabil. 2006;8(5):547-554.

30. Edwards RR, Kronfli T, Haythornthwaite JA, Smith MT, McGuire L, Page GG. Association of catastrophizing with interleukin-6 responses to acute pain. Pain. 2008;140(1):135-144. doi:10.1016/j.pain.2008.07.024
31. Zhou YQ, Liu Z, Liu ZH, et al. Interleukin-6: an emerging regulator of pathological pain. $J$ Neuroinflammation. 2016;13(1):141. doi:10.1186/s12974-016-0607-6

32. Ren K, Torres R. Role of interleukin-1beta during pain and inflammation. Brain Res Rev. 2009;60(1):57-64. doi:10.1016/j.brainresrev. 2008.12.020

33. Gomez-Hernandez J, Orozco-Alatorre AL, Dominguez-Contreras M, et al. Preoperative dexamethasone reduces postoperative pain, nausea and vomiting following mastectomy for breast cancer. BMC Cancer. 2010;10:692. doi:10.1186/1471-2407-10-692

34. Wattwil M, Thorn SE, Lovqvist A, Wattwil L, Gupta A, Liljegren G. Dexamethasone is as effective as ondansetron for the prevention of postoperative nausea and vomiting following breast surgery. Acta Anaesthesiol Scand. 2003;47(7):823-827. doi:10.1034/j.13996576.2003.00172.x

35. Grigoras A, Lee P, Sattar F, Shorten G. Perioperative intravenous lidocaine decreases the incidence of persistent pain after breast surgery. Clin J Pain. 2012;28(7):567-572. doi:10.1097/AJP.0b013e $31823 \mathrm{~b} 9 \mathrm{cc} 8$

36. Kim MH, Lee KY, Park S, Kim SI, Park HS, Yoo YC. Effects of systemic lidocaine versus magnesium administration on postoperative functional recovery and chronic pain in patients undergoing breast cancer surgery: a prospective, randomized, double-blind, comparative clinical trial. PLoS One. 2017;12(3):e0173026. doi:10.1371/journal. pone. 0173026
Journal of Pain Research

\section{Publish your work in this journal}

The Journal of Pain Research is an international, peer reviewed, open access, online journal that welcomes laboratory and clinical findings in the fields of pain research and the prevention and management of pain. Original research, reviews, symposium reports, hypothesis formation and commentaries are all considered for publication. The manuscript

\section{Dovepress}

management system is completely online and includes a very quick and fair peer-review system, which is all easy to use. Visit http:// www.dovepress.com/testimonials.php to read real quotes from published authors. 